\title{
A biomimetic absorbent for removal of trace level persistent organic pollutants from water
}

\author{
Huijuan Liu ${ }^{\text {a }}$, Jiuhui Qu ${ }^{\text {a,*, Ruihua Dai }}{ }^{\text {a,b }}$, Jia Ru ${ }^{\text {a,b }}$, Zijian Wang ${ }^{\text {a }}$ \\ ${ }^{a}$ State Key Laboratory of Environmental Aquatic Chemistry, Research Center for Eco-Environmental Sciences, Chinese Academy of Sciences, \\ Beijing 100085, China \\ ${ }^{\mathrm{b}}$ Graduate School of the Chinese Academy of Sciences, Beijing 100039, China
}

Received 25 May 2006; accepted 14 June 2006

Triolein-embedded absorbent was developed and it could remove lipophilic pollutants from water effectively.

\begin{abstract}
A novel biomimetic absorbent containing the lipid triolein was developed for removing persistent organic pollutants (POPs) from water. The structural characteristics of the absorbent were obtained by SEM and a photoluminescence method. Under optimum preparation conditions, triolein was perfectly embedded in the cellulose acetate (CA) spheres, the absorbent was stable and no triolein leaked into the water. Dieldrin, endrin, aldrin and heptachlor epoxide were effectively removed by the CA-triolein absorbent in laboratory batch experiments. This suggests that $\mathrm{CA}$-triolein absorbent may serve as a good absorbent for those selected POPs. Triolein in the absorbent significantly increased the absorption capacity, and lower residual concentrations of POPs were achieved when compared to the use of cellulose acetate absorbent. The absorption rate for lipophilic pollutants was very fast and exhibited some relationship with the octanol-water partition coefficient of the analyte. The absorption mechanism is discussed in detail.
\end{abstract}

(C) 2006 Elsevier Ltd. All rights reserved.

Keywords: Biomimetic absorbent; Triolein; Organic pollutants; Water treatment

\section{Introduction}

The problem of persistent organic pollutants (POPs) is an urgent global issue requiring immediate attention. These compounds share unique physicochemical characteristics that make them highly mobile and capable of long-range atmospheric transport (Xu et al., 2004). Due to their persistent and lipophilic nature, POPs tend to bioaccumulate in aquatic organisms such as fish and whales and ultimately in humans (Petty et al., 1995). Methods to remove residual POPs that have been released into the environment have attracted increasing attention in recent years (Jones and Voogt, 1999). Most POPs are lipophilic chemicals and this explains their low concentrations in water, usually varying between

\footnotetext{
* Corresponding author. Tel.: +86 1062849151 ; fax: +86 1062923558 . E-mail address: jhqu@ rcees.ac.cn (J. Qu).
}

nanogram and picogram quantities per liter (Peter and Della, 2003; Zhang et al., 2003). Therefore, POPs are especially difficult to remove from water.

The photocatalytic oxidation of POPs on semiconductor surfaces of compounds such as $\mathrm{TiO}_{2}$ and $\mathrm{WO}_{3}$ has recently attracted widespread interest (Balasubramanian et al., 2004) and ozonation has been proposed as a potential alternative. These latest methods were shown to be effective in eliminating POPs from water. However, the cost of photocatalytic oxidation and ozonation for such purposes, especially for removing trace levels of POPs from water, needs to be further ascertained to ensure their competitiveness. Adsorption by activated carbon is widely used in removing organic pollutants from water as shown in many relevant studies (Urano et al., 1999; Miyake et al., 2003; Bembnowska et al., 2003). However, due to the non-selectivity of activated carbon to all organic pollutants and its tendency to desorption after saturation, it is difficult to remove trace or ultratrace POPs near the 
environmental levels by adsorption on active carbon. Some studies have shown that activated carbon is not useful for organic halogens with a concentration below $5 \mu \mathrm{g} \mathrm{L}^{-1}$ (Wang and Liu, 2001). This has stimulated interest in the development of new adsorbents to remove low concentrations of organic contaminants from water.

Even at trace concentrations in water, POPs can accumulate in organisms. Many studies have been carried out on bioaccumulation in diverse organisms such as white whales (Andersen et al., 2001), arctic wolves (Mary and Birgit, 1999), South African fur seals (Walter et al., 1999), marketable fish (Ahmed and Aly, 2004) and mussels (Azza et al., 2004; Perihan and Hulya, 2004). Semipermeable membrane devices (SPMD), which are also based on the diffusion of hydrophobic substances from water to membrane bags filled with lipophilic phases, are widely used for monitoring organic contaminants in water (Huckins et al., 1990; Frank, 2005; Lu and Wang, 2003; Lu et al., 2002).

Using the concept of bioaccumulation, a novel composite absorbent containing the lipid triolein is proposed to remove trace POPs from water. The absorbent was prepared by embedding triolein into cellulose acetate (CA) spheres. Triolein has a high accumulation capacity $\left(10^{5}-10^{7}\right)$ for trace concentrations of POPs in water (Chiou, 1985) and it exhibits low membrane solubility and permeability because of its large molecular mass of 885.45 Da (Huckins et al., 1990). A cellulose acetate $(\mathrm{CA})$ polymer was chosen for preparing hybrid materials because it can be easily molded into different forms such as membranes, fibers and spheres. Furthermore, its hydrophilicity improves the accessibility of aqueous solutions to the surface of the film. The biomimetic absorbent was prepared with these pollution-free and environmentally friendly raw materials and the spherical absorbent was easy to use in water treatment processes.

This paper addresses the optimum preparation method, the characteristics of the absorbent and the efficient removal of some representative POPs from water. The absorption mechanism is also discussed.

\section{Experimental section}

\subsection{Materials}

Cellulose acetate was purchased from the Chemical Reagent Corporation (Shanghai, China), Triolein (98\% purity) was purchased from the Jinlong Chemical Reagent Corporation (Beijing, China), and Tween 80 (A.R.), acetone (A.R.), $\mathrm{Mg}\left(\mathrm{ClO}_{4}\right)_{2}$ (A.R.) and liquid olefin were purchased from the Chemical Reagent Corporation (Beijing, China).

The reagents n-hexane, dichloromethane and methanol, purchased from Fisher Scientific Company (USA), were all pesticide grade. The target analytes dieldrin, aldrin, endrin and heptachlor epoxide (99.0\% purity) were purchased from the Environmental Protection Center of the Chinese Department of Agriculture. An SPE-filtration device with a vacuum pump and ten connectors, SPE units, and 500-mg C18 cartridges were supplied by Agilent Corporation (USA).

\subsection{Preparation of absorbent}

The preparation method is presented in a patent (Liu et al., 2004) and briefly described here. A solution of CA in acetone was prepared and varying amounts of triolein, water, $\mathrm{Mg}\left(\mathrm{ClO}_{4}\right)_{2}$ and Tween 80 were then added to the solution. The mixtures were kept at $35^{\circ} \mathrm{C}$ for 2 days and stirred in order to admix uniformly. Thus, viscous syrups with different triolein loadings were carefully prepared and homogeneous phases were obtained. Each viscous syrup was added drop by drop to a glass tube containing the liquid olefin (about $2 / 3$ of the volume). The temperature ranges of the CA solution and liquid olefin were kept at $30-35^{\circ} \mathrm{C}$ and $15-20{ }^{\circ} \mathrm{C}$ respectively. During the process of the viscous syrup dripping through the air to the column of liquid olefin and through the action of surface tension, a white, spherical absorbent was formed which collected at the bottom of the glass tube. Finally, composite absorbents were washed with distilled water to remove all soluble impurities. The TOC of the leachate was determined using a Phoenix 8000 Total Organic Carbon Analyzer (Tekmar-Dohrmann Co., USA). After changing the water up to 10 times over a 3-day period, the TOC level of the leachate was near that of distilled water.

The water, dissolved in the casting solution, was used as a pore forming agent. As mentioned previously by Meier et al. (2004), water acts as a nonsolvent but it can form porous membranes. An additional advantage of adding water is to enhance the density of the casting solution so that it can drop into liquid olefin and form spheres. In the present study the influence of water on the absorbent was followed in detail. Optimum results were obtained with $12-14 \%(\mathrm{w} / \mathrm{w})$ water in the casting solution. Under these conditions the spheres of absorbent are readily formed in liquid olefin. The amount of triolein was another important factor affecting the formation of spheres in liquid olefin because the triolein had an oily consistency. A $2-5 \%(\mathrm{w} /$ w) triolein concentration in the casting solution was found to be an appropriate range. Under these conditions a smooth surface absorbent was easily prepared.

Cellulose acetate spheres were also prepared using the above method but without adding triolein. All of the absorbents were stored in distilled water before use.

\subsection{Analytical methods}

Scanning electron micrographs (SEM) of the surface and the cross sections of freeze-dried absorbents were obtained using a Hitachi S-3000N scanning electron microscope. Samples were prepared, frozen in liquid nitrogen and then coated with gold. The triolein concentration in the water that leaked out from the CA-triolein absorbent was analyzed using a Hitachi F-3000 fluorescence spectrometer.

A gas chromatograph (HP-6890N) equipped with a ${ }^{63} \mathrm{Ni}$ electron capture detector (GC-ECD) and a HP-5 fused silica capillary column $(30 \mathrm{~m} \times$ $0.32 \mathrm{~mm} \times 0.25 \mu \mathrm{m}$ ) was used to determine the residual concentration of the studied POPs. The temperature of the injector and the detector was kept at $250{ }^{\circ} \mathrm{C}$ and $300^{\circ} \mathrm{C}$, respectively. Column temperature was programmed at $85^{\circ} \mathrm{C}$, increasing at $10^{\circ} \mathrm{C} \min ^{-1}$ to $180^{\circ} \mathrm{C}$, and then increasing at $20^{\circ} \mathrm{C}$ $\min ^{-1}$ to $280^{\circ} \mathrm{C}$, and held for $20 \mathrm{~min}$ at $280^{\circ} \mathrm{C}$. Nitrogen of extra purity (>99.999\%) was used as the carrier gas. The flow rate was $2.5 \mathrm{~mL} \mathrm{~min}{ }^{-1}$ and the pressure was kept at 20 psi. Data were collected and analyzed with Agilent Chem-Station.

\subsection{Absorption experiment}

The four organics selected to test the effectiveness of the novel absorbent were dieldrin, aldrin, endrin and heptachlor epoxide. Absorption experiments were carried out in triplicate with two types of absorbents (CA absorbent and $\mathrm{CA}-$ triolein absorbent) in batch mode. A fresh solution was prepared with distilled water free of the detectable analytes listed above throughout the course of the absorption experiment. Distilled water $(100 \mathrm{~mL})$ in conical flasks was spiked with an organic standard and agitated for $8 \mathrm{~h}$ using a horizontal shaker operated $170 \mathrm{rpm}$ at $25^{\circ} \mathrm{C}$ to dissolve the organics in water homogeneously. One gram of absorbent was employed in contact with a solution of $100 \mathrm{~mL}$. The absorption experiments were carried out in conical flasks with agitation provided by a shaker at $170 \mathrm{rpm}$. The temperature was controlled at $25^{\circ} \mathrm{C}$ by an air bath. The initial concentration of selected organics was $10 \mu \mathrm{g} \mathrm{L}^{-1}$. Samples were withdrawn at regular intervals for analysis. Finally, the water samples were concentrated by SPE (C-18) and analyzed by gas chromatography for residual organics in the samples. 


\section{Results and discussion}

\subsection{Characteristics of the absorbent}

$\mathrm{CA}$ as a rigid thermoplastic is soluble in acetone and can form a smooth and defect-free film. SEM images of the surface of $\mathrm{CA}$ absorbent and $\mathrm{CA}-$ triolein absorbent revealed a relatively smooth surface, free from obvious cracks, holes, or other defects and no triolein exposed on the surface of the absorbent.

Fig. 1 presents SEM micrographs of the absorbent crosssections containing no triolein and those containing $2.0 \%$ (w/w) triolein. Fig. 1(a) and (c) show that both absorbents presented a very thin layer from the top surface. Triolein droplets cannot be seen in the layer that should be the CA membrane. This could be explained by the aggregating rate of $\mathrm{CA}$ into the air being faster than that of triolein. In addition, triolein loading is likely to affect the process of formation of the CA membrane. A careful inspection of the images of Fig. 1 (a) and (c) reveals that the layer of the $\mathrm{CA}$-triolein absorbent was thinner than that of CA. The thickness of the CA and CA-triolein absorbent layers was about $10 \mu \mathrm{m}$ and $5 \mu \mathrm{m}$, respectively.

Fig. 1 (b) and (d) are the partially amplified images of Fig. 1 (a) and (c), respectively. Careful inspection reveals that CA formed mesh structures. The image of the CA-triolein absorbent shows fewer pores and triolein droplets that have been embedded into the hole formed by the CA fiber. Huo et al. (2005) showed that CA was immiscible with triolein in the triolein/CA membrane. In this absorbent, triolein was wrapped in the thin CA polymer, and constituted a significant fraction of the lipid pool in the absorbent. This biomimetic absorbent should be most effective in its capacity to accumulate lipophilic substances. In addition, the thin CA polymer can prevent triolein leakage.

\subsection{Absorption efficiency for four selected analytes}

Table 1 presents the physicochemical properties of the test POPs. Although dieldrin, endrin, aldrin and heptachlor epoxide are all non-polar compounds, they have several physicochemical dissimilarities which may affect their CA membrane permeation rates and steady state $\mathrm{CA}$-triolein-water distribution coefficients.

Fig. 2 shows residual concentrations of the four selected chemicals in test water as a function of time. The data suggest that the removal rates of $\mathrm{CA}$ and $\mathrm{CA}$-triolein absorbents were very fast at the beginning and slowed down gradually later. Nearly $90 \%$ of the aldrin was removed after $1 \mathrm{~h}$ of absorption by both absorbents. At the end of the experiment, the concentrations in the residual water treated with $\mathrm{CA}$ and $\mathrm{CA}-$ triolein absorbents were 0.43 and $0.21 \mu \mathrm{g} \mathrm{L}^{-1}$, respectively. Lower residual concentrations of the four analytes were observed following treatment with $\mathrm{CA}$-triolein compared to the $\mathrm{CA}$ absorbent over a $24-\mathrm{h}$ absorption time period.

The removal of hydrophobic compounds by the CA-triolein absorbent may be attributed to both absorption to cellulose acetate and uptake by triolein. The results also confirm that the presence of triolein in the absorbent significantly increased the accumulation of strongly hydrophobic POPs. $\mathrm{Xu}$ et al. (2005) showed that strongly hydrophobic compounds having high values of $\log K_{\mathrm{ow}}$ have a much greater tendency to accumulate in the triolein phase than in the CA phase.

Our results reveal a higher affinity of CA and CA-triolein absorbents for the most hydrophobic and less polar compounds of the group. The role of triolein is probably similar to that of an organic solvent in a liquid-liquid extraction. The distribution of a neutral organic compound between the biomimetic absorbent and water can be correlated with the


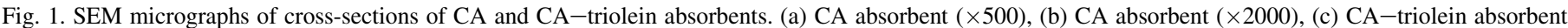
containing $2.0 \%(\mathrm{w} / \mathrm{w})$ triolein $(\times 500)$, and $(\mathrm{d}) \mathrm{CA}-$ triolein absorbent containing $2.0 \%(\mathrm{w} / \mathrm{w})$ triolein $(\times 2000)$. 
Table 1

Selected physicochemical properties of the test organochlorine compounds

\begin{tabular}{lllll}
\hline Compound & $\begin{array}{l}\text { Molecular } \\
\text { weight }\end{array}$ & $\begin{array}{l}\text { Water solubility } \\
\left(\mathrm{mg} \mathrm{L}^{-1}, 25^{\circ} \mathrm{C}\right)\end{array}$ & Log $K_{\mathrm{ow}}$ \\
\hline Aldrin & 365 & 0.027 & 5.663 & (Brás et al., 1999) \\
Dieldrin & 381 & 0.195 & 5.48 & (Mackay, 1982) \\
Heptachlor epoxide & 389.2 & 0.35 & 4.51 & (Xu et al., 2005) \\
Endrin & 381 & 0.25 & 4.56 & (Mackay, 1982) \\
\hline
\end{tabular}

octanol-water partition coefficient. Values of $\log K_{\mathrm{ow}}$ for this group of compounds range from 5.663 (aldrin) to 4.51 (heptachlor epoxide). The highest residual concentration of absorption obtained for heptachlor epoxide can perhaps be explained by its smallest octanol-water partition coefficient.

The stability of the CA-triolein absorbent was evaluated by measuring triolein in water by a photoluminescence method. Water samples were withdrawn at regular times from the blank experiments. No leakage of triolein was detected. Huo et al. (2005) also showed that when triolein was added to the CA syrup, it tended to fill the holes that had formed in the process of phase transition and the low triolein content did not affect the structure of the CA membrane. In this paper, a $2 \%(\mathrm{w} / \mathrm{w})$ triolein loading did not affect the structure of the CA membrane surface. This was also confirmed by SEM images and the absorbent was stable in water.

\subsection{Absorption mechanism}

The absorption process by the absorbent can be described in four consecutive steps:

1. transport in the bulk of the solutions;
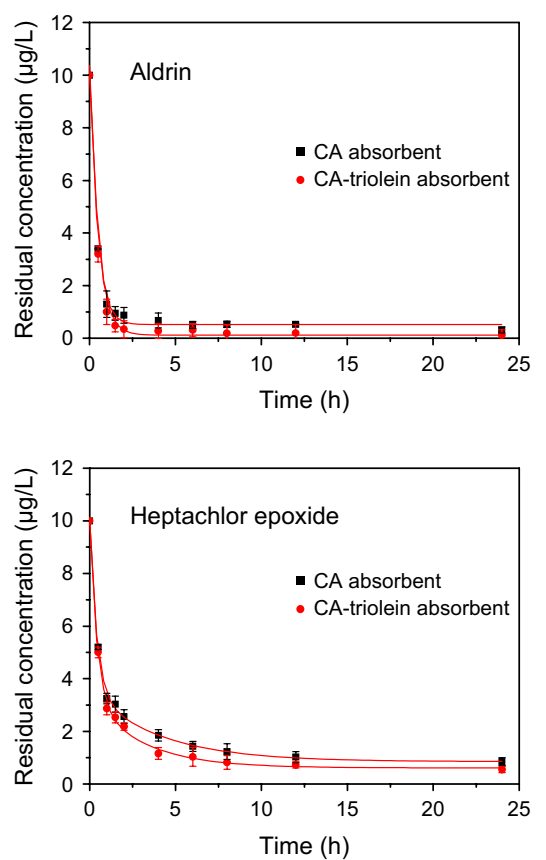

2. diffusion across the liquid film surrounding the absorbent particles;

3. molecular diffusion in the liquid contained in the pores and in the adsorbate along the pore walls;

4. sorption and desorption within the particle and on the external surface.

Any of the four previous steps or any combination of these steps may be the rate controlling factor(s). Transport in the solution is sometimes rate determining in large-scale field processes. Our experimental sorption systems were designed to eliminate the effect of transport in the solution by rapid mixing so that this could not be the rate-limiting step.

According to Weber and Morris (1963), a plot of solute sorbed against the square root of contact time should yield a straight line passing through the origin, if the rate limiting step is intra-particle diffusion. The most widely applied intra-particle diffusion equation for bio-sorption systems is given by Weber and Morris (1963) as:

$q_{t}=k \cdot t^{0.5}$

where $q_{t}$ is the amount of adsorbate at time $t\left(\mu \mathrm{g} \mathrm{g}^{-1}\right)$; and $k$ is the rate constant $\left(\mu \mathrm{g}\left(\mathrm{g} \mathrm{h}^{0.5}\right)^{-1}\right)$.

Fig. 3 shows plots of $q_{t}$ vs. $t^{0.5}$ in an intra-particle diffusion model using the absorption results by different absorbents for the four analytes. They indicate that the $q_{t}$ vs. $t^{0.5}$ relationship is multi-linear. Table 2 shows the $k$ values for the four compounds. It is obvious that the order of absorption rate for the four analytes follows the order $k_{1}>\mathrm{k}_{2}>k_{3}$.

Previous studies (Wu et al., 2001) also showed that such plots may present a multi-linearity confirming that two or more steps occur in the absorption process. The first sharper
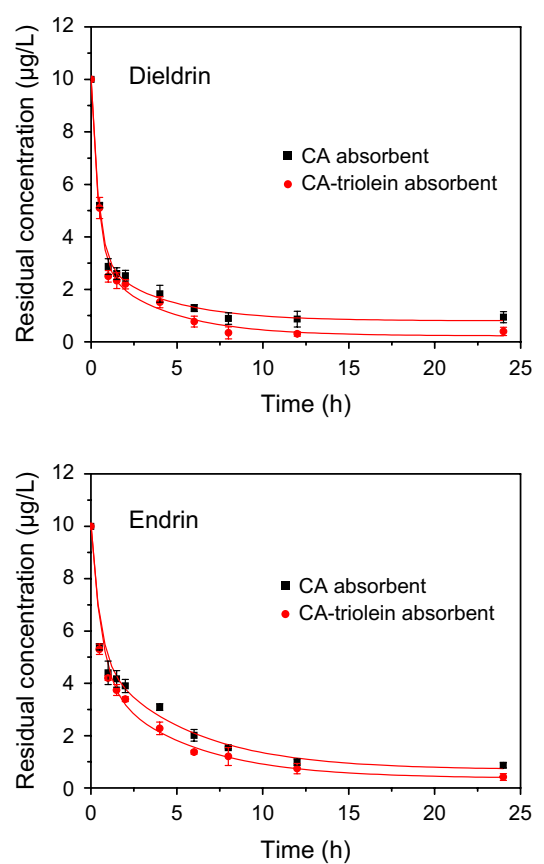

Fig. 2. Residual concentration of four selected analytes after absorption by $\mathrm{CA}$ and $\mathrm{CA}-$ triolein absorbents as a function of time. $V=0.1 \mathrm{~L} ; T=25^{\circ} \mathrm{C}$; $N=170 \mathrm{rpm}$; absorbent: $1 \mathrm{~g} ; C_{0}=10 \mu \mathrm{g} \mathrm{L}{ }^{-1}$; error bars shown are standard deviations. 

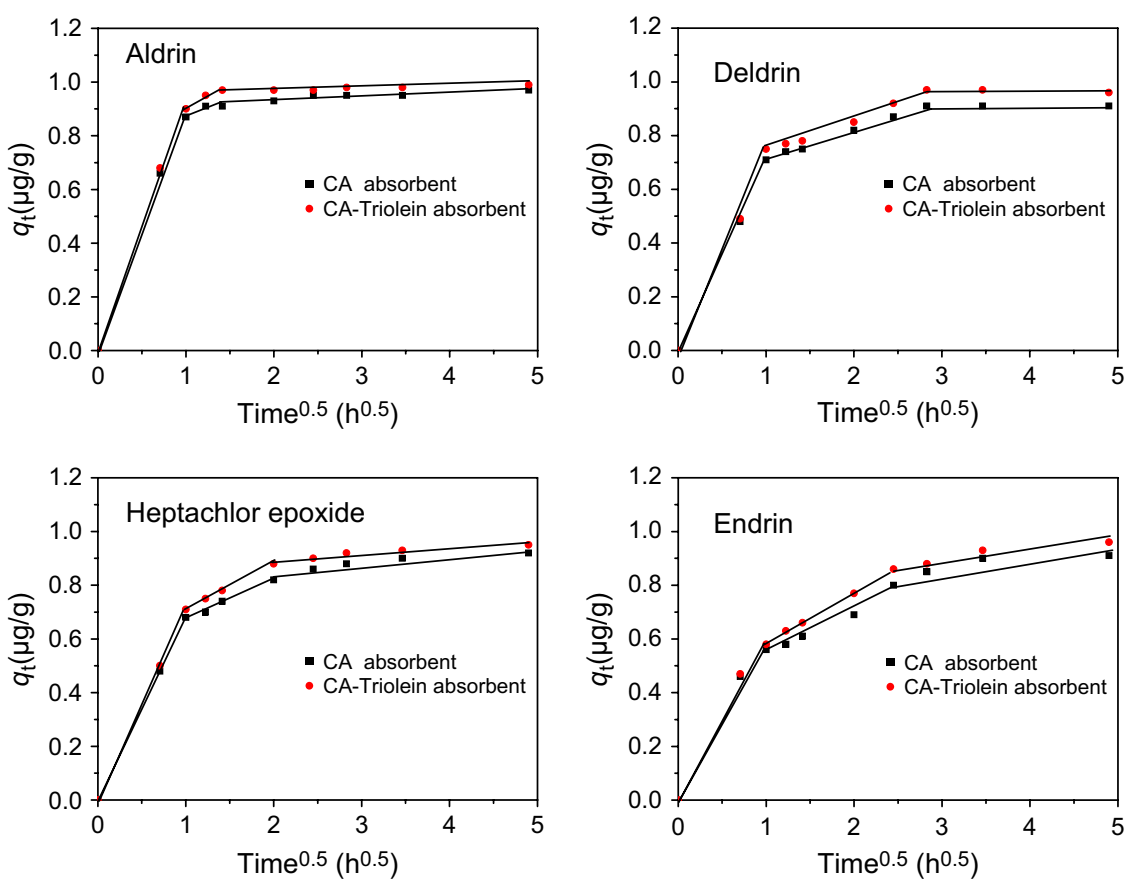

Fig. 3. Plot of $q_{t}$ vs. $t^{0.5}$ of the four selected analytes after absorption by CA and CA-triolein absorbents.

portion describes the external surface absorption or instantaneous absorption stage. The second portion is the gradual absorption stage, where the intra-particle diffusion is ratecontrolled. The third linear portion corresponds to the final equilibrium stage, where the intra-particle diffusion starts to slow down due to the extremely low solute concentration in solution.

The results of absorption experiments could be explained from the structure of absorbents. These absorbents had the same outer surface of CA and triolein as the major adsorptive material was embedded into CA. Organic pollutants were firstly adsorbed onto the surface of absorbents, and then transferred into channels of CA, and finally absorbed into the triolein. At the beginning of the absorption process, the driving force is the organic pollutant concentration difference between the external surface of CA and CA-triolein absorbents and the surrounding fluid concentration. There was the same concentration difference across the boundary layer around each particle, indicating the same driving force. In this stage the outer surface of the absorbent plays the dominant role in the absorption process.

With prolonged time, organic pollutants that were adsorbed on the surface of absorbents gradually transferred into the CA channels. We hypothesize that the diffusion of compounds through the CA membrane was the rate-limiting step in the uptake of test pollutants by the CA-triolein absorbent. This hypothesis is based on the following rationale. First, the diffusion of non-electrolyte organic compounds through some synthetic polymers was reported to occur slowly. Second, the interfacial tension between the corresponding lipidmembrane phases within the absorbent is obviously much lower after and the transport of analytes across this interface should be even more rapid. Thus, organic pollutants in CA channels can rapidly transfer into triolein. The transfer speed is accelerated with increasing $\log K_{\mathrm{ow}}$ of the organic pollutants, accordingly leaving more adsorptive positions in CA. Hence, the rate-limiting step appears to be the time required for the test compounds to diffuse through the CA membrane.

The absorption rate for the four selected POPs has some relation with the $\log K_{\text {ow }}$ of the chemicals. The $\log K_{\text {ow }}$ values of dieldrin and aldrin are larger than those of heptachlor epoxide and endrin, resulting in the absorption rates of dieldrin and aldrin being faster than those of heptachlor epoxide and endrin. Endrin and heptachlor epoxide have similar log $K_{\text {ow }}$ but there is little difference in their absorption rates. This may be due to the different molecular weights and shapes of

Table 2

Rate of absorption on different absorbents for the four analytes at different adsorptive stages

\begin{tabular}{|c|c|c|c|c|c|c|}
\hline \multirow[t]{2}{*}{ Analyte } & \multicolumn{2}{|l|}{$K_{1}$} & \multicolumn{2}{|l|}{$K_{2}$} & \multicolumn{2}{|l|}{$K_{3}$} \\
\hline & CA absorbent & $\begin{array}{l}\text { CA-triolein } \\
\text { absorbent }\end{array}$ & CA absorbent & $\begin{array}{l}\text { CA-triolein } \\
\text { absorbent }\end{array}$ & CA absorbent & $\begin{array}{l}\text { CA-triolein } \\
\text { absorbent }\end{array}$ \\
\hline Aldrin & 0.8912 & 0.9206 & 0.0352 & 0.0265 & 0.0146 & 0.0056 \\
\hline Dieldrin & 0.7016 & 0.7321 & 0.1092 & 0.1221 & 0.0039 & 0.0069 \\
\hline Heptachlor epoxide & 0.6766 & 0.7122 & 0.1286 & 0.1507 & 0.0237 & 0.0170 \\
\hline Endrin & 0.5902 & 0.5956 & 0.1626 & 0.1727 & 0.0085 & 0.0222 \\
\hline
\end{tabular}


the two types of molecules. Previous studies (Huckins et al., 1990, 1993) have shown that the sampling rate of lipids by an organic phase was determined on a molecular basis with respect to steric factors such as molecular mass and size, shape, rotational freedom and flexibility.

\section{Conclusion}

The SEM results of the CA-triolein absorbent suggest that triolein was effectively embedded into CA spheres and that a thin film of CA surface layer containing no triolein was formed, thus ensuring that virtually no triolein leaked out through the CA membrane.

The initial results from this new absorbent are promising for the removal of non-polar compounds. The CA-triolein absorbent can efficiently and quickly accumulate hydrophobic POPs from water. Lower residual concentrations of selected POPs were obtained by the CA-triolein absorbent in comparison with the $\mathrm{CA}$ absorbent.

These encouraging results will help in future work to determine absorption isotherms, to elucidate the absorption mechanisms involved, and to address engineering issues for implementing the process on a larger scale.

\section{Acknowledgements}

The authors appreciate the generous financial support of this work from the National Natural Science Foundation of China $(50578154,20337020)$ and the National Science Fund for Distinguished Young Scholars (50225824).

\section{References}

Ahmed, E.N., Aly, M.A.A., 2004. Organochlorine contamination in some marketable fish in Egypt. Chemosphere 54, 1401-1406.

Andersen, G., Kovacs, K.M., Lydersen, C., Skaare, J.U., Gjertz, I., Jenssen, B.M., 2001. Concentrations and patterns of organochlorine contaminants in white whales (Delphinapterus leucas) from Svalbard, Norway. The Science of the Total Environment 264, 267-281.

Azza, K., Ahmed, E.N., Tarek, O.S., Amany, E.S., Aly, M.A.A., 2004. Polychlorinated biphenyls and chlorinated pesticides in mussels from the Egyptian Red Sea coast. Chemosphere 54, 1407-1412.

Balasubramanian, G., Dionysiou, D.D., Suidan, M.T., Baudin, I., LaÌné, J.M., 2004. Evaluating the activities of immobilized $\mathrm{TiO}_{2}$ powder films for the photocatalytic degradation of organic contaminants in water. Applied Catalysis B: Environmental 47, 73-84.

Bembnowska, A., Pelech, R., Milchert, E., 2003. Absorption from aqueous solutions of chlorinated organic compounds onto activated carbon. Journal of Colloid and Interface Science 265, 276-282.

Brás, I.P., Santos, L., Alves, A., 1999. Organochlorine pesticides removal by pinus bark sorption. Environmental Science and Technology 33, 631-634.

Chiou, C.T., 1985. Partition coefficients of organic compounds in lipid-water systems and correlations with fish bioconcentration factors. Environmental Science and Technology 19, 57-62.

Frank, S.L., 2005. Review of passive accumulation devices for monitoring organic micropollutants in the aquatic environment. Environmental Pollution 136, 503-524.
Huckins, J.N., Tubergen, M.W., Manuweera, G.K., 1990. Semipermeable membrane devices containing model lipid: a new approach to monitoring the bioavailability of lipophilic contaminants and estimating their bioconcentration potential. Chemosphere 20, 533-552.

Huckins, J.N., Manuweera, G.K., Petty, J.D., Mackay, D., Lebo, J.A., 1993. Lipidcontaining semipermeable membrane devices for monitoring organic contaminants in water. Environmental Science and Technology 27, 2489-2496.

Huo, J.X., Liu, H.J., Qu, J.H., Wang, Z.J., Ge, J.T., Liu, H.N., 2005. Preparation and characteristic of triolein-embedded composite sorbents for water purification. Separation and Purification Technology 44, 37-43.

Jones, K.C., Voogt, P., 1999. Persistent organic pollutants (POPs): state of the science. Environmental Pollution 100, 209-211.

Liu, H.J., Qu, J.H., Dai, R.H., Ru, J., 2004. China Patent, 200410062266.5.

Lu, Y.B., Wang, Z.J., Huckins, J., 2002. Review of the background and application of $\mathrm{CA}$-triolein semipermeable membrane devices in aquatic environmental study. Aquatic Toxicology 60, 139-153.

Lu, Y.B., Wang, Z.J., 2003. Accumulation of organochlorinated pesticides by CA-triolein semipermeable membrane device (triolein-SPMD) and rainbow trout. Water Research 37, 2419-2425.

Mackay, D., 1982. Correlation of bioconcentration factors. Environmental Science and Technology 16, 274-278.

Mary, G., Birgit, M.B., 1999. Contaminant residue levels in arctic wolves (Canis lupus) from the Yukon Territory, Canada. The Science of the Total Environment 243, 329-338.

Meier, M.M., Kanis, L.A., Soldi, V., 2004. Characterization and drug-permeation profiles of microporous and dense cellulose acetate membranes: influence of plasticizer and pore forming agent. International Journal of Pharmaceutics 278, 99-110.

Miyake, Y., Sakoda, A., Yamanashi, H., Kaneda, H., Suzuki, M., 2003. Activated carbon absorption of trichloroethylene (TCE) vapour stripped from TCE-contaminated water. Water Research 37, 1852-1858.

Perihan, B.K., Hulya, B.O., 2004. A survey to determine levels of chlorinated pesticides and PCBs in mussels and seawater from the Mid-Black Sea Coast of Turkey. Marine Pollution Bulletin 48, 1076-1083.

Peter, K.K.L., Della, W.S., 2003. A preliminary investigation of persistent organic pollutants in ambient air in Hong Kong. Chemosphere 52, 1397-1403.

Petty, J.D., Huckins, J.N., Orazio, C.E., Lebo, J.A., Poulton, B.C., Gale, R.W., Charbonneau, C.S., Kaiser, E.M., 1995. Determination of waterborne bioavailable organochlorine pesticide residues in the lower Missouri River. Environmental Science and Technology 29, 2561-2566.

Urano, K., Yamamoto, E., Tonegawa, M., Fujie, K., 1999. Absorption of chlorinated organic compounds on activated carbon from water. Water Research 25, 1459-1464.

Walter, V., Marion, W., Elke, S., Bernd, L., Herbert, O., 1999. Levels of organochlorines (DDT, PCBs, toxaphene, chlordane, dieldrin, and HCHs) in blubber of South African fur seals (Arctocephalus pusillus pusillus) from Cape Cross/Namibia. Marine Pollution Bulletin 38, 830-836.

Wang, Z.S., Liu, W.J., 2001. Drinking Water Treatments for Micropollutant Water Resources. China Architecture \& Building Press, Beijing, p. 62.

Weber, W.J., Morris, J.C., 1963. Kinetics of absorption on carbon from solution. Journal of the Sanitary Engineering Division ASCE 89, 31-60.

Wu, F.C., Tseng, R.L., Juang, R.S., 2001. Kinetic modelling of liquid-phase absorption of reactive dyes and metal ions on chitosan. Water Research 35, 613-618.

Xu, D.D., Zhong, W.K., Deng, L.L., Chai, Z.F., Mao, X.Y., 2004. Regional distribution of organochlorinated pesticides in pine needles and its indication for socioeconomic development. Chemosphere 54, 743-752.

Xu, Y.P., Wang, Z.J., Ke, R.H., Khan, S.U., 2005. Accumulation of organochlorine pesticides from water using triolein embedded cellulose acetate membranes. Environmental Science and Technology 39, $1152-1157$.

Zhang, Z.L., Hong, H.S., Zhou, J.L., Huang, J., Yu, G., 2003. Fate and assessment of persistent organic pollutants in water and sediment from Minjiang River Estuary, Southeast China. Chemosphere 52, 1423-1430. 